\title{
Letter
}

\section{Reappraisal of Variable-Range Hopping in Quantum-Dot Solids}

Arjan J. Houtepen, Daan Kockmann, and Danie\#I Vanmaekelbergh

Nano Lett., 2008, 8 (10), 3516-3520 • DOI: 10.1021/nl8020347 • Publication Date (Web): 13 September 2008

Downloaded from http://pubs.acs.org on January 14, 2009
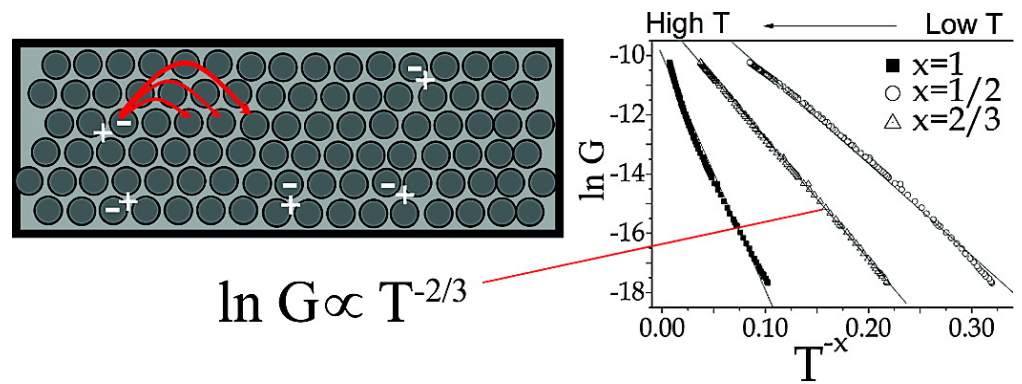

\section{More About This Article}

Additional resources and features associated with this article are available within the HTML version:

- Supporting Information

- Access to high resolution figures

- $\quad$ Links to articles and content related to this article

- Copyright permission to reproduce figures and/or text from this article

\section{View the Full Text HTML}




\title{
Reappraisal of Variable-Range Hopping in Quantum-Dot Solids
}

\author{
Arjan J. Houtepen, ${ }^{, t, \neq}$ Daan Kockmann, ${ }^{\dagger, \S}$ and Daniël Vanmaekelbergh ${ }^{\dagger}$ \\ Condensed Matter and Interfaces, Debye Institute, Utrecht University, PO Box 80.000, \\ 3508 TA Utrecht, The Netherlands, and Optoelectronic Materials, Faculty of Applied \\ Sciences, Delft University of Technology, Julianalaan 136, \\ 2628 BL Delft, The Netherlands
}

Received July 10, 2008; Revised Manuscript Received August 18, 2008

\begin{abstract}
The temperature dependence of the electrical conductivity of assemblies of $\mathrm{ZnO}$ nanocrystals, studied with an electrochemically gated transistor is very accurately described by the relation $\ln \sigma=\ln \sigma_{0}-\left(T_{0} / T\right)^{x}$ with $x=2 / 3$ over the entire temperature range from 7 to $200 \mathrm{~K}$, independent of charge concentration and dielectric environment. These results cannot be explained by existing models but are supported by results on Au nanocrystals where an identical temperature dependence was observed (Zabet-Khosousi et al., Phys. Rev. Lett. 2006, 96 (15), 156403). We propose an adaptation of the Efros-Shklovskii variable-range hopping model by introducing an expression for nonresonant tunneling based on local energy fluctuations, which yields exactly the temperature dependence that is observed experimentally.
\end{abstract}

Films of semiconductor nanocrystals (NCs) constitute a novel class of solid-state materials. Such so-called quantumdot solids hold promise for electrical and opto-electronic devices such as field-effect transistors, ${ }^{2}$ biosensors, ${ }^{3}$ photodetectors, ${ }^{4}$ solar cells, ${ }^{5-8}$ and lasers. ${ }^{9,10}$ There is a large and growing control over the assembly of nanocrystals into single component, ${ }^{11}$ binary, ${ }^{12}$ and even ternary ${ }^{13}$ structures that will be used in such devices. In addition, there is a large effort in device-oriented research involving quantum-dot solids, especially those made of $\mathrm{CdSe},{ }^{5,14} \mathrm{PbSe},{ }^{2,6} \mathrm{PbS},{ }^{4,15}$ and $\mathrm{ZnO}^{16,17}$ nanocrystals. It is clear that for any real application electron transport through films of NCs has to be understood. In addition to the technological interest in quantum-dot solids, they are of fundamental interest for physics, since they form a new class of electron conducting materials in which the occupation of the quantum-dot energy levels and their electronic interaction can be engineered..$^{18,19}$

The energy landscape of an array of nanocrystals is inherently disordered. Small differences in size, shape, and position of the nanocrystals lead to variations in the energies of electrons (or holes) occupying the quantum-confined orbitals. In such disordered systems, charge transport occurs via hopping. As first noted by Mott, a hopping step does

* To whom correspondence should be addressed. E-mail: a.j.houtepen@ tudelft.nl.

$\dagger$ Utrecht University.

$¥$ Delft University of Technology.

$\S$ Current address: MESA+, University of Twente, PO Box 217, 7500 AE Enschede, The Netherlands. not necessarily take place between nearest-neighbor hoppingsites, but may span a greater distance, that is, variable-range hopping, VRH. ${ }^{20}$

The temperature dependence of electronic conductivity forms a key characteristic in the study of transport in any system (superconductor, metal, inorganic semiconductor, molecular conductor). In the case of nanocrystal arrays belonging to the class of disordered systems, the temperature dependence of conductivity is inherently related to the disorder in the electronic structure. More precisely, it results from (i) the hopping rate $\Gamma(\Delta E, R, T)$ as a function of energy mismatch $\Delta E$ and distance $R$ between the donor and acceptor site, and temperature $T$ and (ii) the statistical relation between the distance and the energy mismatch between two hopping sites, $R(\Delta E)$, the latter being a disorder parameter specific for a given material. Item (i), the hopping rate, is usually assumed to obey the Miller-Abrahams expression ${ }^{21}$

$$
\Gamma(\Delta E, R, T)=\Gamma_{0} \exp \left(-\frac{2 R}{a}-\frac{\Delta E}{k_{\mathrm{B}} T}\right)
$$

Here $a$ is the localization length, which represents the decay of the wave function in the tunnel barrier between the hopping sites (i.e., between the nanocrystals).

Regarding item (ii), which is directly related to the densityof-states $g(E)$ near the Fermi level, several models have been put forward. Mott assumed that the density of states is constant as a function of energy (i.e., $g(E)=g_{0}$ ) and showed that in that case $R(\Delta E)=3 / 4\left(4 / 3 \pi g_{0} \Delta E\right)^{-1 / 3} \cdot{ }^{22}$ Later Efros and Shklovskii showed that when Coulomb interactions between different hopping sites are important, $g(E)$ is depleted near the Fermi level. This effect is called the 
Coulomb gap; it changes item (ii) to $R(\Delta E)=$ $e^{2}\left(4 \pi \varepsilon \varepsilon_{0} \Delta E\right)^{-1} .{ }^{23}$ At every temperature, there is an optimum combination of $R$ and $\Delta E$ where $\Gamma(\Delta E, R, T)$ is maximum. The hopping rates at this value of $\Delta E$ form the dominant contribution to the total current. ${ }^{24}$ If $R(\Delta E)$ is known, it can be inserted in eq 1 and the maximum in $\Gamma(\Delta E, T)$ can be found by identifying the root in the function $\mathrm{d} \Gamma(\Delta E, T) / \mathrm{d} \Delta E$. In the case of Mott-VRH, this yields a hopping rate given by $\ln \Gamma \propto T^{-1 / 4}$, which results in a conductivity $\sigma$ with the same $T$ dependence. ${ }^{22}$ The Coulomb gap in the EfrosShklovskii model results in a different $T$ dependence of the form $\ln \sigma \propto T^{-1 / 2} .{ }^{23}$

The temperature-dependent electronic conductivity in disordered atomic solids has been studied extensively. ${ }^{25}$ In contrast, such studies on quantum-dot solids are limited and the results are not yet consistent. Yu et al. ${ }^{26}$ and Wehrenberg et al. ${ }^{27}$ studied the temperature dependence of electrochemically charged $\mathrm{CdSe}$ and $\mathrm{PbSe}$ nanocrystal assemblies, respectively, in a temperature range of $\sim 10$ to $\sim 150 \mathrm{~K}$. They concluded that the conductivity has a $\ln \sigma \propto T^{-1 / 2}$ dependence. Talapin and Murray studied the same PbSe NC system using a field-effect transistor (FET) setup and found $\ln \sigma \propto$ $T^{-1 / 4}$. $^{2}$ Finally, Mentzel et al. recently presented more elaborate FET measurements on PbSe quantum-dot solids and concluded that the charge transport shows simple Arrhenius behavior, i.e. $\ln \sigma \propto T^{-1}{ }^{28}$ It should be remarked here that the determination of the $T$ dependence of the conductivity requires a high-quality data set and is prone to significant errors.

Here we present detailed results on electron transport in a $\mathrm{ZnO}$ quantum-dot solid at various charge concentrations. We chose this well-studied system because it is chemically stable and can be charged with a variable number of conduction electrons, between 0 and 6 per quantum dot. ${ }^{18,29-31}$ The quality of our data sets is such that the temperature dependence can be determined accurately; that is, it can be shown that the conductivity is very accurately described by the relation $\ln \sigma=\ln \sigma_{0}-\left(T_{0} / T\right)^{x}$ with $x=2 / 3$ over the whole temperature range from 7 to $200 \mathrm{~K}$, independent of charge concentration or dielectric environment. It should be remarked that the $T$ dependence that we find for $\mathrm{ZnO} \mathrm{NC}$ assemblies has also been reported for transport in assemblies of Au NCs. ${ }^{1}$ This strongly points to the existence of a welldefined transport law.

We reconsider nonresonant electron transfer, that is, basis (i) of the variable range hopping model, while basis (ii), that is, $R(\Delta E)$, remains unchanged. We take into account the thermal broadening of each of the energy levels of the disordered system and present an equation for the transfer rate analogous to those provided by Hopfield ${ }^{32}$ and Marcus. ${ }^{33}$ This equation replaces the conventional Miller-Abrahams term in the VRH models. If we do so, we find that the Mott $T^{-1 / 4}$ law remains nearly unchanged, while the exponent of the Efros-Shklovskii model changes from $1 / 2$ to $2 / 3$. The adapted ES VRH model quantitavely explains our results obtained with $\mathrm{ZnO}$ quantum-dot solids as well as previously reported results for assemblies of Au NCs. ${ }^{1}$ Our model for
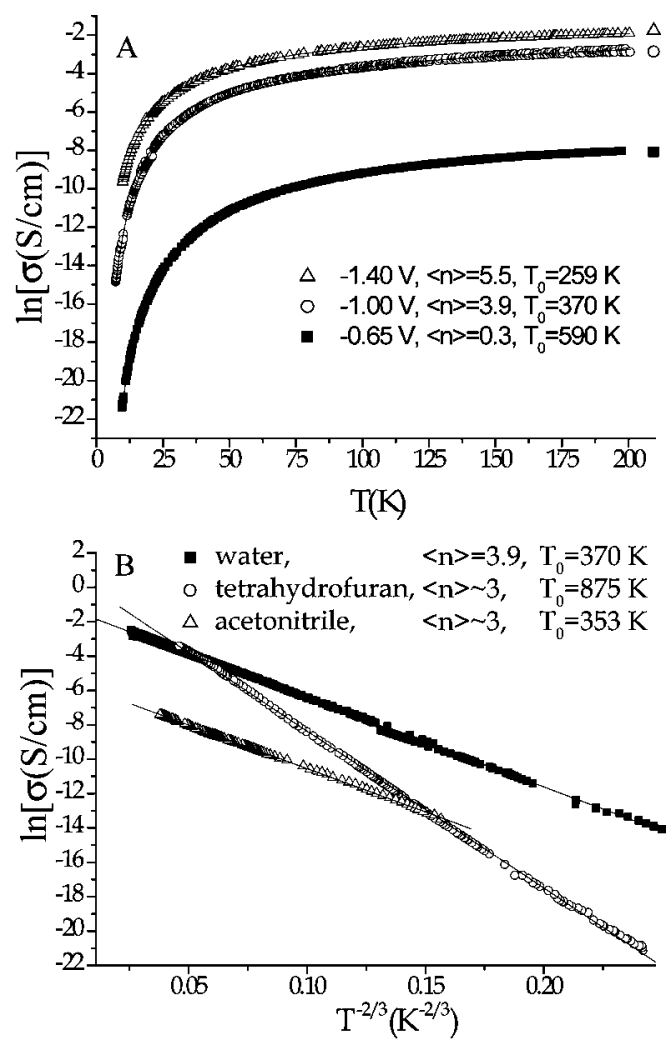

Figure 1. (A) The natural logarithm of the conductivity as a function of $T$ of $\mathrm{ZnO}$ QD solids in phosphate buffer at three different charge concentrations. The solid lines are fits to eq 2. (B) The natural logarithm of the conductivity of three different $\mathrm{ZnO}$ QD assemblies in electrolytes with different solvents, plotted as a function of $T^{-2 / 3}$.

nonresonant electron transfer opens new avenues for understanding electron conduction in disordered systems.

Assemblies of $\mathrm{ZnO}$ nanocrystals were dropcast on homemade interdigitated gold-arrays. This system was used as a working electrode in an electrochemical cell, which enabled us to vary the orbital occupation per nanocrystal over a large range. A silicon diode (Lakeshore SD670-B) was integrated into the cell to provide accurate temperature measurements. The number of injected conduction electrons per nanocrystal was monitored optically. ${ }^{19,34}$ Different film-permeating electrolytes were used to obtain charge compensation of the injected electrons: ${ }^{29}$ nitrogen-purged phosphate buffer $(\mathrm{pH}$ 8.0), $0.1 \mathrm{M} \mathrm{LiClO}_{4}$ in acetonitrile (ACN), and $0.1 \mathrm{M} \mathrm{LiClO}_{4}$ in tetrahydrofuran (THF). Charging and discharging was completely reversible up to $\sim 6$ conduction electrons per NC. At a given charge density, the system was frozen, and the linear electronic conductance was measured as a function of $T$. The temperature was scanned from the melting point of the electrolyte to $7 \mathrm{~K}$ and back. Extensive details on the experimental procedures can be found in the Supporting Information and in ref 31.

The temperature dependence of electronic conductivity was measured at different charge concentrations, in different electrolytes and for quantum dots of different diameters. Figure 1A shows the conductivity of an assembly of $3.6 \mathrm{~nm}$ ZnO QDs in phosphate buffer, between 7 and $200 \mathrm{~K}$, at charge densities $\langle n\rangle$ of 0.3 and 3.9 conduction electrons per 
quantum dot and of a $3.3 \mathrm{~nm}$ QD assembly at $\langle n\rangle=5.5$. The solid lines in Figure 1A are fits to

$$
\ln \sigma=\ln \sigma-\left(\frac{T_{0}}{T}\right)^{x}
$$

This expression is typical for conductivity in disordered insulators. An exponent $x$ of $1 / 4,1 / 2$, or 1 corresponds to Mott VRH, ES VRH, and nearest-neighbor hopping (NNH), respectively. In the fits of Figure $1 \mathrm{~A}$ (solid lines) $\sigma_{0}, T_{0}$ and $x$ were free-fitting parameters. The exponent $x$ was $0.654 \pm$ 0.001 for $\langle n\rangle=0.3,0.651 \pm 0.003$ for $\langle n\rangle=3.9$, and $0.665 \pm$ 0.003 for $\langle n\rangle=5.5$. The absolute value of the conductivity is higher at higher $\langle n\rangle$, while the constant $T_{0}$ is lower, but it is clear that the exponent in eq 2 is independent of charge concentration. Note that the exponent is very close to the factor $2 / 3$. Figure 1B shows $\ln \sigma$ as a function of temperature in different electrolytes, plotted on a $T^{-2 / 3}$ scale. The curves in Figure 1B were chosen because they were obtained at comparable charge concentrations. Since $\langle n\rangle$ is similar it is justified to compare the values of $T_{0}$. We found that $T_{0}$ is similar for water and acetonitrile $(\sim 360 \mathrm{~K})$, but that it is significantly larger for tetrahydrofuran $(875 \mathrm{~K})$.

All curves in Figure 1 show the conductivity for both decreasing and increasing temperature. The two scanning directions are indistinguishable. This is an indication of the stability of the frozen sample and the reliability of the measured temperature. The exact determination of the exponent in eq. 2 requires absolute care. We, therefore, analyze our results in several ways and show that the exponent is very close to $2 / 3$ over the entire temperature range and that previously applied models with exponents of $1 / 4,1 / 2$, or 1 are not valid. Figure $2 \mathrm{~A}$ shows a logarithmic conductivity data set on three different temperature scales: $T^{-1}, T^{-1 / 2}$, and $T^{-2 / 3}$. From this figure it is clear that $x=2 / 3$ is the only acceptable fit to the data set. We must conclude that Mott VRH, nor ES VRH, nor NNH can explain the measured conductivity over the full temperature range.

To the best of our knowledge no existing model predicts an exponent of $2 / 3$ but, surprisingly, an exponent of 0.65 was found recently by Zabet-Khosousi et al. for electronic conductivity in disordered films of Au NCs. ${ }^{1}$ The authors remark that an exponent between $1 / 2$ and 1 may result from a transition of ES VRH to NNH. This was also the explanation given by $\mathrm{Yu}$ et al. $^{26}$ for the temperature dependence of conductivity in CdSe $\mathrm{NC}$ assemblies. A transition from $x=1 / 2$ to $x=1$ is attributed to "saturation" of variable-range hopping: as the temperature increases the average hopping distance decreases until nearest-neighbor hopping results. In the ES VRH model, the temperature at which this transition occurs depends on the dielectric constant and the localization length. ${ }^{25}$ It should, therefore, be different in different electrolytes and at different charging levels. As a result, the exponent in eq. 2 , when fitted over the whole $T$ range, should vary. However, in all our experiments the determined exponent was $2 / 3$, independent of dielectric environment (i.e., electrolyte solvent) and charge concentration. In addition, we did not observe a change in $T$ dependence between low and high temperatures.

An elaborate analysis is presented in Figure 2B,C. If, in a certain $T$ range, a single exponent $x$ describes the conduc-
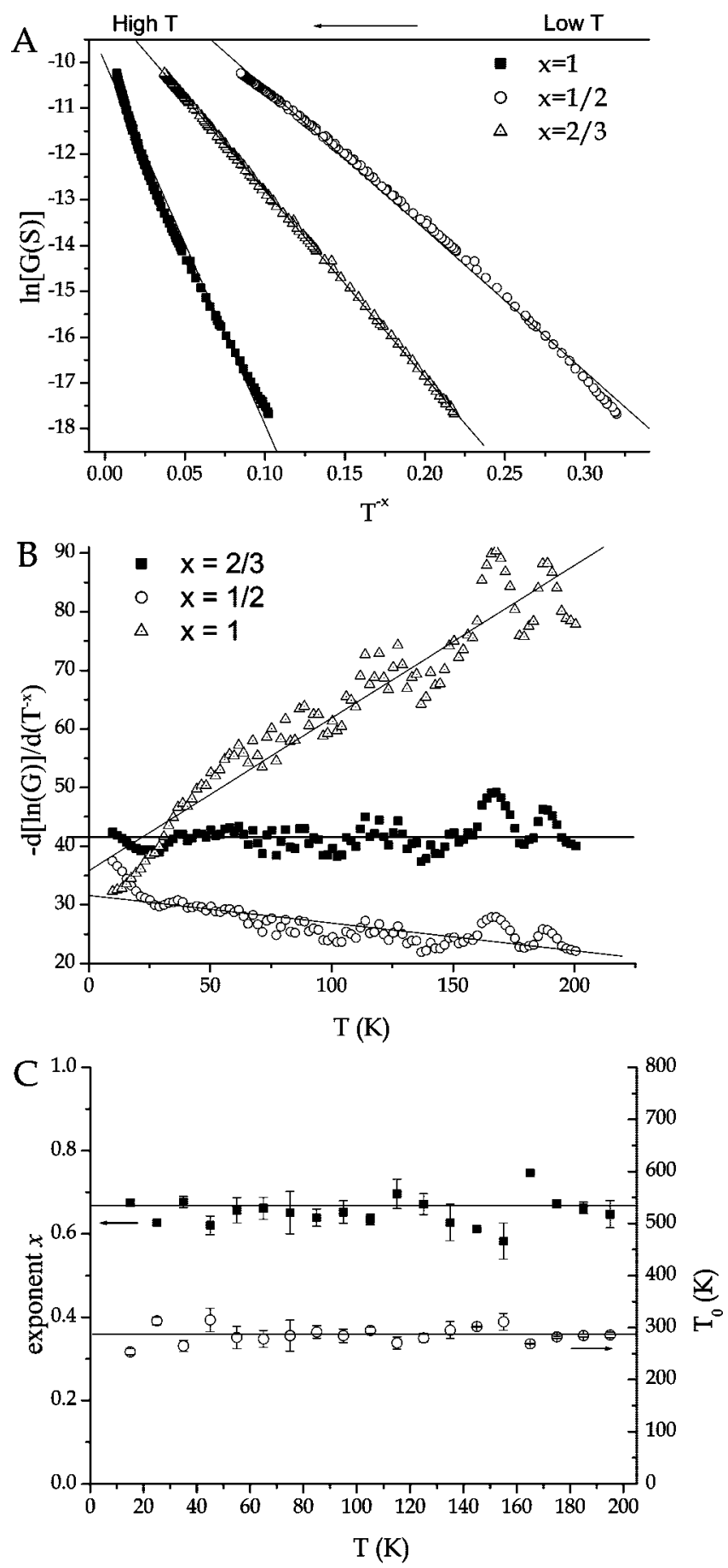

Figure 2. (A) Logarithmic conductance of an assembly of $3.3 \mathrm{~nm}$ ZnO QDs in phosphate buffer at $\langle n\rangle=5.5$, plotted on different temperature scales. (B) The derivative of the logarithmic conductance with respect to $T^{-1 / 2}$ (open circles), $T^{-2 / 3}$ (solid squares), and $T^{-1}$ (triangles). This last curve was divided by 2 for clarity. Solid lines are linear fits. (C) The fitted value of the exponent (solid squares) and $T_{0}$ (open circles) in eq 2 in temperature bins of $10 \mathrm{~K}$. The values of $x$ and $T_{0}$ obtained for the full temperature range are shown as solid lines.

tance $G$ well, the plot of $\ln G$ vs $T^{-x}$ should be straight and the derivative of this curve should be constant. A transition from $x=1 / 2$ to $x=1$ should show up as a deviation from this constant value at the transition temperature. Figure 2B shows $\mathrm{d}(\ln G) / \mathrm{d}\left(T^{-x}\right)$ for different values of $x$. The curves with $x=1 / 2$ and $x=1$ are not constant at any temperature, 
while the curve with $x=2 / 3$ is constant over the entire $T$ range. Another way to detect a possible transition between different exponents is to fit the conductivity separately in different temperature ranges (Figure 2C). The temperature is divided into $10 \mathrm{~K}$ bins, and the exponent is determined by fitting these bins to eq 2 . In order to obtain reliable fits in such a small $T$ range the constant $\sigma_{0}$ is fixed to the value obtained by fitting the full data set. Both the exponent and $T_{0}$ determined in this way are quite constant; there is no systematic deviation from $x=2 / 3$. From the above analyses, it is clear that there is no transition between different exponents in the range of temperatures that was investigated.

To explain the conductivity in 2D arrays of Ag NCs Remacle et al. presented a model in which VRH at around the Fermilevel coexists with $\mathrm{NNH}$ at much higher energies. ${ }^{35}$ They argued that at some energy well above the Fermi-level there will always be an energy level that is resonant with an energy level of the neighboring hopping site. This hopping channel should result in thermally activated transport. In our experiments the Fermi-level is changed over $750 \mathrm{meV}$ (see Figure 1A) which should lead to a much larger $\mathrm{NNH}$ current in the model of Remacle et al. The total temperature dependence should, therefore, approach $\ln \sigma \propto T^{-1}$. However, even this large change in the Fermi-level did not result in a deviation from $\ln \sigma \propto T^{-2 / 3}$. In addition, we studied samples with nanocrystals of different sizes and, hence, different energy separations between the confined energy-levels; the $T$ exponent $x$ remained $2 / 3$. Note also, that in a completely different system, i.e. assemblies of Au NCs, an identical $T$-dependence was observed. ${ }^{1}$ These results imply that a transport mechanism based on the coexistence of two transport paths is highly unlikely.

As a result of the energetic disorder in quantum-dot solids, there will be an energy-mismatch between a donor site and all acceptor sites in the environment. Tunneling is only possible through the absorption and/or emission of a small amount of energy. In an all-inorganic solid-state material, the energy-mismatch is paid for through interaction with lattice phonons, in organic solid-state materials it is typically via molecular vibrations while in the case of charge transfer between molecules in solution the polarization of the liquid environment plays an important role. Electrochemically gated quantum-dot solids contain contributions from all of the above. Here we do not focus on the microscopic origin, ${ }^{36}$ but use a general thermodynamic approach that describes broadening of each energy level within the density-of-states of the ensemble of nanocrystals due to temporal energy fluctuations. In the Supporting Information, it is shown that these energy fluctuations result in a Gaussian broadening of a given energy level with a Gaussian width that depends on heat capacity and temperature:

$$
g_{\text {fluc }}(E)=g_{0} \exp \left(-\frac{\left(E-E_{0}\right)^{2}}{2 k_{\mathrm{B}} T^{2} C_{\mathrm{v}}}\right)
$$

Here $C_{\mathrm{v}}$ is the heat capacity of the conduction electron, $E_{0}$ is the average energy of that electron, and $g_{\text {fluc }}(E)$ is the time
Table 1. Comparison of the $T$ Dependence of VRH Derived with the Miller-Abrahams Expression (Equation 1) and with Equation 4

\begin{tabular}{|c|c|c|c|c|}
\hline \multirow{2}{*}{$\begin{array}{c}\begin{array}{c}\text { rate } \\
\text { equation }\end{array} \\
\text { model }\end{array}$} & \multicolumn{2}{|c|}{$\begin{array}{c}\text { Miller- } \\
\text { Abrahams }\end{array}$} & \multicolumn{2}{|r|}{ eq 4} \\
\hline & $x$ & $T_{0}$ & $x$ & $T_{0}$ \\
\hline \multirow[t]{2}{*}{ Mott 3D } & $1 / 4$ & 24 & $2 / 7$ & 1.69 \\
\hline & & $\pi a^{3} g_{0} k_{\mathrm{B}}$ & & $a^{3} g_{0} \sqrt{C_{\mathrm{v}} k_{\mathrm{B}}}$ \\
\hline \multirow{2}{*}{$\begin{array}{l}\text { Efros- } \\
\text { Shklovskii }\end{array}$} & $1 / 2$ & $2 e^{2}$ & $2 / 3$ & $3 \sqrt{3} e^{2}$ \\
\hline & & $a 4 \pi \varepsilon \varepsilon_{0} k_{\mathrm{B}}$ & & $\mathrm{a} \pi \varepsilon \varepsilon_{0} \sqrt{C_{\mathrm{v}} k_{\mathrm{B}}}$ \\
\hline
\end{tabular}

averaged-density-of-states of the broadened energy level. This is in agreement with recent scanning-tunnelingspectroscopy studies on nanocrystal quantum-dots that have revealed a Gaussian shape of the energy-levels with a width of tens of meV..$^{36-39}$ The overlap between $g_{\text {fluc }}(E)$ of a donor and an acceptor site in a hopping event, separated by a distance $R$ and an energy mismatch $\Delta E$, determines the rate of nonresonant charge-transfer, ${ }^{40}$ which is given by

$$
\Gamma=\frac{\Gamma_{0}}{2 T \sqrt{2 \pi k_{\mathrm{B}} C_{\mathrm{v}}}} \exp \left(-\frac{2 R}{a}-\frac{(\Delta E)^{2}}{4 k_{\mathrm{B}} T^{2} C_{\mathrm{v}}}\right)
$$

A full derivation can be found in the Supporting Information. Equation 4 is mathematically equivalent to the electron transfer rate derived by Marcus, ${ }^{33}$ with a reorganization energy of $C_{\mathrm{v}} T \ll \Delta E$. In the classical regime, the heat capacity is independent of temperature. This is in accordance with the experimental observation that the Gaussian width $w$ of the broadened level depends linearly on temperature ${ }^{36}$ (from equation 3 it follows that $w^{2}=k_{\mathrm{B}} C_{v} T^{2}$ ). Thus, we use the classical expression $C_{v}=3 k_{\mathrm{B}}{ }^{41}$ The Marcus ${ }^{33}$ and Hopfield ${ }^{32}$ models for electron transfer, as well as eq 4, differ from the Miller-Abrahams model (eq 1) in that they have a Gaussian, rather than an exponential, dependence on energy mismatch.

Equation 4 can be used to derive the $T$ dependence of electronic conductivity using either the Mott or the EfrosShklovskii expression for $R(\Delta E)$. This derivation is outlined in the Supporting Information; the results are shown in Table 1. The well-known Mott $T^{-1 / 4}$ law changes to a $T^{-2 / 7}$ dependence. The difference in the exponent is small; it is unlikely that a clear distinction between the two can be made in an experiment. In contrast, the Efros-Shklovskii $T^{-1 / 2}$ dependence changes to $T^{-2 / 3}$, a difference which is experimentally observable, as was shown above. It can be seen that in the revised models, the expressions for $T_{0}$ are very similar to the classical expressions if $C_{\mathrm{v}} \approx k_{\mathrm{B}}$. We now evaluate the experimental $T_{0}$ values to test the adapted VRH model.

$T_{0}$ in the ES model depends inversely on both the localization length and the dielectric constant $\varepsilon$ of the medium surrounding the hopping sites. Here, $\varepsilon$ was varied through the choice of the electrolyte solvent: $\operatorname{THF}(\varepsilon=7.6)$, $\operatorname{ACN}(\varepsilon=37.5)$, and water $(\varepsilon=78.5)$. Thus, $T_{0}$ is expected to be significantly larger for THF than for the other solvents. Figure 1B shows that this is indeed the case. As a consequence of overall charge neutrality a higher charge concentration corresponds to a higher ionic strength of the 
electrolyte, and, consequently, to a higher $\varepsilon$ of the assembly. Figure 1A clearly shows that $T_{0}$ decreases with increasing $\langle n\rangle$, again in agreement with the prediction of our model.

In the Wentzel-Kramers-Brillouin approximation, the localization length $a$ is defined as $a=2 \hbar\left(m^{*} E_{\text {barrier }}\right)^{-1 / 2}$. With a $\mathrm{ZnO}$ effective electron mass of $m^{*}=0.19 m_{\mathrm{e}}$ and a barrier height $E_{\text {barrier }}$ of $4 \mathrm{eV}$ (estimated from the electrochemical reductionpotential), the localization length is estimated to be $0.63 \mathrm{~nm}$. At the lowest charge concentration of $\langle n\rangle=0.3$, measured in phosphate buffer, this localization length yields a dielectric constant of 67, in good agreement with the expected value for water at this low charge concentration (i.e., low ionic strength).

In conclusion, we have shown that the temperature dependence of conductivity in $\mathrm{ZnO}$ quantum-dot solids is very accurately described by the relation $\ln \sigma=\ln \sigma_{0}-$ $\left(T_{0} / T\right)^{2 / 3}$. This is true for different dielectric environments, at different charge concentrations and for quantum dots of different diameters in the entire $T$ range between 7 and 200 $\mathrm{K}$. Since there is, to the best of our knowledge, no transport mechanism that predicts a temperature-dependence of this form, we propose an adaptation of the Efros-Shklovskii VRH model that can quantitatively explain our results. We replace the conventionally used Miller-Abrahams expression for nonresonant transfer by one that is based on temporal energy fluctuations. As a result, the $T$-dependence of the conductivity in the Mott model remains nearly unchanged, but the $T^{-1 / 2}$ dependence in the Efros-Shklovskii model changes to a $T^{-2 / 3}$ law. We conclude that our experimental results, and possibly many others provided in the literature, ${ }^{1}$ can now be explained with the Efros-Shklovskii variablerange hopping model. Hence, the present model for nonresonant electron transfer opens new avenues for understanding electronic conduction in disordered systems.

Acknowledgment. The authors thank P. Liljeroth and J. J. Kelly for helpful discussions.

Supporting Information Available: Elaborate experimental details and the full mathematical derivation of eqs 3 and 4 as well as the derivation of the variable-range hopping expressions mentioned in the text. This material is available free of charge via the Internet at http://pubs.acs.org.

\section{References}

(1) Zabet-Khosousi, A.; Trudeau, P. E.; Suganuma, Y.; Dhirani, A. A.; Statt, B. Phys. Rev. Lett. 2006, 96 (15), 156403.

(2) Talapin, D. V.; Murray, C. B. Science 2005, 310 (5745), 86-89.

(3) Hansen, J. A.; Wang, J.; Kawde, A. N.; Xiang, Y.; Gothelf, K. V.; Collins, G. J. Am. Chem. Soc. 2006, 128 (7), 2228-2229.

(4) Konstantatos, G.; Howard, I.; Fischer, A.; Hoogland, S.; Clifford, J.; Klem, E.; Levina, L.; Sargent, E. H. Nature 2006, 442 (7099), 180183.

(5) Gur, I.; Fromer, N. A.; Geier, M. L.; Alivisatos, A. P. Science 2005, 310 (5747), 462-465.

(6) Kim, S. J.; Kim, W. J.; Cartwright, A. N.; Prasad, P. N. App. Phys. Lett. 2008, 92 (19), 191107.
(7) Schaller, R. D.; Agranovich, V. M.; Klimov, V. I. Nature Phys. 2005, 1 (3), 189-194.

(8) Trinh, M. T.; Houtepen, A. J.; Schins, J. M.; Hanrath, T.; Piris, J.; Knulst, W.; Goossens, A. P. L. M.; Siebbeles, L. D. A. Nano Lett. 2008, 8 (6), 1713-1718.

(9) Klimov, V. I.; Mikhailovsky, A. A.; Xu, S.; Malko, A.; Hollingsworth, J. A.; Leatherdale, C. A.; Eisler, H. J.; Bawendiz, M. G. Science (Washington, DC) 2000, 290 (5490), 314-317.

(10) Wang, C. J.; Wehrenberg, B. L.; Woo, C. Y.; Guyot-Sionnest, P. J. Phys. Chem. B 2004, 108 (26), 9027-9031.

(11) Murray, C. B.; Kagan, C. R.; Bawendi, M. G. Science 1995, 270 (5240), 1335-1338.

(12) Shevchenko, E. V.; Talapin, D. V.; Kotov, N. A.; O’Brien, S.; Murray, C. B. Nature 2006, 439 (7072), 55-59.

(13) Shevchenko, E. V.; Kortright, J. B.; Talapin, D. V.; Aloni, S.; Alivisatos, A. P. Adv. Mater. 2007, 19 (23), 4183.

(14) Coe, S.; Woo, W. K.; Bawendi, M.; Bulovic, V. Nature 2002, 420 (6917), 800-803.

(15) McDonald, S. A.; Konstantatos, G.; Zhang, S.; Cyr, P. W.; Klem, E. J. D.; Levina, L.; Sargent, E. H. Nat. Mater. 2005, 4 (2), 138-142.

(16) Sun, B.; Sirringhaus, H. Nano Lett. 2005, 5, 2408-2413.

(17) Jin, Y.; Wang, J.; Sun, B.; Blakesley, J. C.; Greenham, N. C. Nano Lett. 2008, 8 (6), 1649-1653.

(18) Roest, A. L.; Kelly, J. J.; Vanmaekelbergh, D.; Meulenkamp, E. A. Phys. Rev. Lett. 2002, 89 (3), 036801.

(19) Yu, D.; Wang, C.; Guyot-Sionnest, P. Science 2003, 300 (5623), 127780.

(20) Mott, N. F. Philos. Mag. 1969, 19 (160), 835-852.

(21) Miller, A.; Abrahams, E. Phys. Rev. 1960, 120 (3), 745 LP-755.

(22) Mott, N. F.; Davis, E. A. Electronic processes in non-crystalline materials, 2nd ed.; Oxford University Press: London, 1979; p 590.

(23) Efros, A. L.; Shklovskii, B. I. J. Phys. C: Solid State Phys. 1975, 8, L49-L51.

(24) Mott, N. F. Rev. Mod. Phys. 1978, 50 (2), 203.

(25) Shklovskii, B. I., Efros, A. L. Electronic properties of doped semiconductors; Springer-Verlag: Berlin, 1984; Vol. 45.

(26) Yu, D.; Wang, C. J.; Wehrenberg, B. L.; Guyot-Sionnest, P. Phys. Rev. Lett. 2004, 92 (21), 216802.

(27) Wehrenberg, B. L.; Yu, D.; Ma, J. S.; Guyot-Sionnest, P. J. Phys. Chem. B 2005, 109 (43), 20192-20199.

(28) Mentzel, T. S.; Porter, V. J.; Geyer, S.; MacLean, K.; Bawendi, M. G.; Kastner, M. A. Phys. Rev. B 2008, 77 (7), 075316.

(29) Roest, A. L.; Houtepen, A. J.; Kelly, J. J.; Vanmaekelbergh, D. Faraday Disc. 2004, 125, 55-62.

(30) Vanmaekelbergh, D.; Houtepen, A. J.; Kelly, J. J. Electrochim. Acta 2007, (53), 1140-1149.

(31) Houtepen, A. J. Charge injection and transport in quantum confined and disordered systems. Utrecht University, 2007.http://igiturarchive. library.uu.nl/dissertations/2007-0626-203806/index.htm.

(32) Hopfield, J. J. Proc. Natl. Acad. Sci. U.S.A. 1974, 71 (9), 3640-3644.

(33) Marcus, R. A. Angew. Chem., Int. Ed. Eng. 1993, 32 (8), 1111-1121.

(34) Houtepen, A. J.; Vanmaekelbergh, D. J. Phys. Chem. B 2005, 109 (42), 19634-19642.

(35) Remacle, F.; Beverly, K. C.; Heath, J. R.; Levine, R. D. J. Phys. Chem. B 2003, 107 (50), 13892-13901.

(36) Jdira, L.; Overgaag, K.; Stiufiuc, R.; Grandidier, B.; Delerue, C.; Speller, S.; Vanmaekelbergh, D. Phys. Rev. B 2008, 77 (20), 205308.

(37) Bakkers, E. P. A. M.; Hens, Z.; Zunger, A.; Franceschetti, A.; Kouwenhoven, L. P.; Gurevich, L.; Vanmaekelbergh, D. Nano Lett. 2001, 1 (10), 551-556.

(38) Liljeroth, P.; van Emmichoven, P. A. Z.; Hickey, S. G.; Weller, H.; Grandidier, B.; Allan, G.; Vanmaekelbergh, D. Phys. Rev. Lett. 2005, 95 (8), 86801.

(39) Jdira, L.; Liljeroth, P.; Stoffels, E.; Vanmaekelbergh, D.; Speller, S. Phys.l Rev. B 2006, 73 (11), 115305.

(40) Chandler, R. E.; Houtepen, A. J.; Nelson, J.; Vanmaekelbergh, D. Phys. Rev. B 2007, 75 (8), 085325.

(41) Kittel, C. Introduction to solid state physics, 7th ed.; John Wiley \& Sons: New York, 1996.

NL8020347 